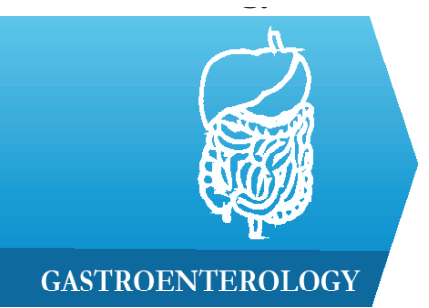

1) Department of Medicine, Kasturba Medical College, Manipal Academy of Higher Education, Manipal, India

2) Department of Microbiology, Kasturba Medical College, Manipal Academy of Higher Education, Manipal, India

3) Department of Biostatistics, Manipal Academy of Higher Education, Manipal, India

\title{
Spectrum of bacterial infections and predictors of mortality in adult cirrhotic patients
}

\author{
Chandrashekar Udyavara Kudru¹, Vandana Kalwaje Eshwara², \\ Shivashankara Kaniyoor Nagiri ${ }^{1}$, Vasudeva Guddattu ${ }^{3}$
}

\begin{abstract}
Background. Bacterial infections are more frequent in patients with decompensated cirrhosis than those with compensated liver disease and account for significant morbidity and mortality in them. Once an infection develops, it induces excessive production of pro-inflammatory cytokines leading to organ failure and death.
\end{abstract}

Aims. This study aims to identify the clinical characteristics and outcome of bacterial infections affecting various organ systems in patients with liver cirrhosis and to determine factors associated with mortality.

Methods. A cross sectional study was performed on subjects with cirrhosis having microbiologically proven bacterial infection involving various organ systems, admitted to a tertiary care hospital in southern India. Demographic, clinical data, laboratory parameters and outcome details were noted. Univariate associations and subsequent multivariate logistic regression analysis was performed to determine factors associated with mortality.

Results. The study included 158 patients. Chronic alcohol intake was the most common etiology of cirrhosis $(66.4 \%)$. Community acquired infections occurred more frequently than hospital acquired infections ( $85.5 \%$ vs $14.5 \%)$. The common site of isolation of etiological agent was ascitic fluid (38.3\%) followed by blood $(24.3 \%)$, respiratory tract $(15.5 \%)$ and urinary tract $(14.5 \%)$. Gram negative bacterial infections were more common (74.3\%), Escherichia coli being the most frequent pathogen (38.5\%). Mortality was noted in $38(24 \%)$ patients. The factors associated with mortality were the type of infection, Child Pugh category, acute kidney injury, hepatic encephalopathy, urinary tract infection, and creatinine and bilirubin levels. Multivariate logistic regression analysis revealed that type of infection (OR: 0.33, 95\% CI: 0.11-1.01), ascitic fluid infection (OR: 2.81, 95\% CI: 1.11-7.12), hepatic encephalopathy (OR: $0.17,95 \%$ CI: 0.070-0.422) and acute kidney injury (OR: 0.19, $95 \%$ CI: 0.077-0.502) were significantly associated with in-hospital mortality.

Conclusion. This study indicates that the type of infection, hepatic encephalopathy, ascitic fluid infection and acute kidney injury are associated with mortality in cirrhotic patients. Early effective treatment and prevention of these complications may help modify the outcome.

Keywords: acute kidney injury, ascitic fluid infection, bacterial infections, Child Pugh score, hepatic encephalopathy

\section{Introduction}

Despite the advances in medical care, bacterial infections remain very common in patients with liver cirrhosis and account for significant morbidity and mortality in them $[1,2]$. Infections are more frequent in patients with decompensated cirrhosis than in those with compensated liver disease. The impaired immune responses such as decreased phagocytic activity, neutrophil dysfunction, decreased complement levels in serum and an impaired opsonic activity both in ascitic fluid and serum increase the susceptibility of cirrhotic patients to bacterial infections [3-5].

Spontaneous bacterial peritonitis (SBP), urinary tract infection (UTI), pneumonia and bacteremia are the commonly described bacterial infections among patients with liver disease [6,7]. 
The majority of the bacterial infections in cirrhotic patients are caused by Gram-negative bacteria, whereas gram positive bacteria comprise about $20 \%$ and anaerobes only $3 \%$ of these infections [8].

Once the infection occurs, excessive production of pro-inflammatory cytokines in cirrhosis further facilitates the development of serious complications such as shock, hepatic encephalopathy, multiple organ failure and death $[1,4,5]$.

Hence, early recognition of the infection and proper management is essential in order to minimize the complications and reduce mortality. We designed this study to identify the clinical characteristics and outcome of bacterial infections affecting various organ systems in patients with cirrhosis and to study the distribution and outcome of bacterial infections in relation to the severity of liver dysfunction as per Child Pugh classification.

\section{Materials and methods}

This was a cross sectional study conducted at a tertiary care hospital in Southern India. Only one admission of the patient during the study period was considered. Subjects aged $\geq 18$ years with cirrhosis having microbiologically proven bacterial infection involving various organ systems were admitted to the hospital and those with culturenegative neutrocytic ascites (CNNA) were included. We excluded patients with human immunodeficiency virus (HIV) infection, secondary bacterial peritonitis and those on cancer chemotherapy and steroid therapy. The study protocol was approved by the Institutional Ethics Committee and an informed consent was taken from all the subjects.

The diagnosis of cirrhosis was based on the clinical and biochemical features suggestive of chronic liver disease. Abdominal ultrasound was performed in all the cases. A detailed history including symptoms indicative of bacterial infection was obtained from each patient. They were subjected to physical examination to detect the presence of fever, anemia, jaundice, ascites and encephalopathy. Blood, urine, ascitic fluid, sputum, endotracheal tube and wound cultures were sent according to site of infection.

\section{Definitions}

Community acquired infection was defined as infection present upon hospitalization or diagnosed within the first 48 hours of admission to the hospital. Infection occurring after 48 hours of admission to the hospital was considered as hospital acquired [9]. SBP was defined as an ascitic fluid infection with PMN count $>250$ cells/ $\mathrm{mm}^{3}$ and positive ascitic fluid culture without evidence of intra-abdominal surgically treatable source of infection. Culture-negative neutrocytic ascites (CNNA) was defined as an ascitic fluid infection with PMN count $>250$ cells/ $\mathrm{mm}^{3}$ and negative ascitic fluid culture. Monomicrobial nonneutrocytic bacterascites (MMBA) was defined as an ascitic fluid PMN count $<250$ cells $/ \mathrm{mm}^{3}$ with a positive culture for a single organism [10].

The diagnosis of UTI was made if the patient had at least one of the following symptoms or signs: fever $\left(>38^{\circ} \mathrm{C}\right)$, urgency, frequency of micturition, dysuria, or suprapubic tenderness and positive urine culture that were $>10^{5}$ microorganisms per $\mathrm{cm}^{3}$ with no more than two species of microorganisms. Asymptomatic bacteriuria was defined as growth of bacteria $>10^{5}$ microorganisms per $\mathrm{cm}^{3}$ of urine with no more than two species of microorganisms in the absence of urinary symptoms [11]. Respiratory tract infection (RTI) were diagnosed by positive sputum/ endotracheal culture with or without chest X-ray findings. Bacteremia was defined as the presence of bacteria in blood as evidenced by positive blood culture [12]. Acute kidney injury (AKI) was defined as increased serum creatinine $>1.5$ times from the baseline or decreased urine output of $<0.5 \mathrm{ml} / \mathrm{kg} /$ hour for 6 hours. Severity of liver dysfunction was graded using Child Pugh's classification [13]. The patient outcome was defined as survivors or non-survivors during the particular hospital stay.

\section{Statistical analysis}

Categorical variables are summarized by frequency and percentage. Continuous variables are summarized using mean and standard deviation (for normally distributed variables) or median and inter quartile range (for nonnormally distributed variables). Chi-square test was used as test of association between two categorical variables. Independent sample $\mathrm{t}$ test or Mann Whitney $\mathrm{U}$ test was used to compare means across binary variable. Univariate analysis was performed to identify factors associated with mortality. Variables with p-value cutoff of $<0.2$ on univariate analysis were considered for logistic regression analysis. Among the significant variables in the univariate analysis, independent variables with a sample size of $>5$ in each category were included in the multivariate analysis. Forward selection criteria were used to select significant variables in multiple logistic regression analysis for predicting the mortality in cirrhotic patients. A p-value of $<0.05$ is considered statistically significant. Statistical analysis was carried out using EZR plugin in R software version 3.4.1.

\section{Results}

A total of 158 patients formed the study group with 143 (90.5\%) males and 15 (9.5\%) females. The most affected age group was 41-60 years, with 50 patients (31.6\%) being in the age group 41-50 years and 53 patients $(33.5 \%)$ in the age group 51-60 years. The mean age of the patients was $50.97 \pm 10.3$ years. The etiology of cirrhosis was alcoholic liver disease in $105(66.4 \%)$, viral hepatitis in 27 (17\%), cryptogenic in $18(11.4 \%)$, autoimmune diseases in $4(2.5 \%)$ and combined HBV infection and alcoholic liver disease in $3(1.8 \%)$ cases. Co-infection by HBV and $\mathrm{HCV}$ was found in 1 case. 
Table I. Distribution of infections according to Child Pugh class.

\begin{tabular}{|c|c|c|c|c|c|}
\hline \multirow{2}{*}{$\begin{array}{l}\text { Type of infection } \\
(\mathrm{n}, \%)\end{array}$} & \multirow{2}{*}{$\begin{array}{c}\text { Child } \\
\text { Class A }\end{array}$} & \multirow{2}{*}{$\begin{array}{l}\text { Child } \\
\text { Class B }\end{array}$} & \multirow{2}{*}{$\begin{array}{l}\text { Child } \\
\text { Class C }\end{array}$} & \multicolumn{2}{|c|}{ Acquisition, n (\%) } \\
\hline & & & & Community & Hospital \\
\hline AFI $(74,38.3 \%)$ & $1(1.3 \%)$ & $12(16.2 \%)$ & $61(82.4 \%)$ & $68(92 \%)^{*}$ & $6(8 \%)$ \\
\hline Bacteremia $(48,24.3 \%)$ & $3(6.1 \%)$ & $14(28.6 \%)$ & $31(65.3 \%)$ & $41(85.4 \%)$ & $7(14.6 \%)$ \\
\hline RTI $(31,15.5 \%)$ & $1(3.2 \%)$ & $10(32.2 \%)$ & $20(64.5 \%)$ & $21(67.7 \%)$ & $10(32.3 \%)$ \\
\hline UTI $(28,14.5 \%)$ & $2(7.1 \%)$ & $14(50 \%)$ & $12(42.9 \%)$ & $23(82 \%)$ & $5(18 \%)$ \\
\hline Others $(12,6.2 \%)$ & - & - & - & $12(100 \%)$ & - \\
\hline
\end{tabular}

*Only 29 are culture confirmed infections; AFI: Ascitic fluid infection; RTI: Respiratory tract infection; UTI: Urinary tract infection.

Table II. Common bacterial agents isolated from various infections.

\begin{tabular}{|c|c|c|c|c|c|c|}
\hline Bacterial etiology & Total (n) & AFI & Bacteremia & RTI & UTI & Others \\
\hline Escherichia coli & 57 & 15 & 22 & 2 & 17 & 1 \\
\hline Klebsiella pneumoniae & 27 & 2 & 9 & 9 & 4 & 3 \\
\hline Acinetobacter spp. & 13 & 2 & 1 & 9 & 0 & 1 \\
\hline Pseudomaonas aeruginosa & 5 & 1 & 0 & 4 & 0 & 0 \\
\hline Enterobacter spp. & 3 & 0 & 1 & 1 & 0 & 1 \\
\hline Aeromonas spp. & 2 & 2 & 0 & 0 & 0 & 0 \\
\hline MRSA & 6 & 1 & 4 & 0 & 0 & 1 \\
\hline MSSA & 7 & 1 & 2 & 2 & 1 & 1 \\
\hline Enterococcus spp. & 10 & 2 & 1 & 1 & 5 & 1 \\
\hline BHS & 8 & 3 & 5 & 0 & 0 & 0 \\
\hline AHS & 3 & 1 & 2 & 0 & 0 & 0 \\
\hline Streptococcus pneumoniae & 1 & 0 & 0 & 1 & 0 & 0 \\
\hline Mycobacterium tuberculosis & 11 & 0 & 0 & 7 & 1 & 3 \\
\hline
\end{tabular}

MRSA- Methicillin resistant Staphylococcus aureus; MSSA- Methicillin sensitive Staphylococcus aureus; BHS- Beta hemolytic streptococci, AHS- Alpha hemolytic streptococci; AFI: Ascitic fluid infection; RTI: Respiratory tract infection; UTI: Urinary tract infection.

The most common presenting symptoms were abdominal distension 107 (67.7\%) followed by jaundice $90(56.9 \%)$, fever $75(47.4 \%)$ and altered sensorium 45 (28.4\%). Other symptoms included upper gastrointestinal (UGI) bleeding, abdominal pain and cough, which were noted in $20(12.7 \%), 19(12 \%)$ and $20(12.7 \%)$ patients respectively.

Ascitic fluid infection (AFI) (38.3\%) and bacteremia $(24.3 \%)$ comprised majority of infections. Other infections included abscess, lower limb cellulitis and tuberculous cervical lymphadenitis. Out of 74 patients with ascitic fluid infections, 45 had CNNA, 19 had SBP and 10 had MMBA. Among 48 episodes of bacteremia, the source was detected as intra-abdominal in 15, UTI in 3 and RTI in 2 patients, while the source was not detected in 24 cases. Almost $63.9 \%$ of the patients were in Child class C, $32.2 \%$ in Child class B and 3.7\% in Child class A. All types of infections except UTI occurred largely in Child class C, while UTI was predominantly seen in Child class B (Table I). Among the complications, AKI was most common which was observed in 37,13 and 28 patients with AFI,
UTI and bacteremia respectively followed by hepatic encephalopathy which was noted in 21 patients with AFI and 17 cases with bacteremia.

\section{Microbiological spectrum}

Community acquired infections were more frequent than hospital acquired infections [120 (76\%) vs $38(24 \%)]$. Among the community acquired infections, AFI was most common $(n=68,41.4 \%)$ followed by bacteremia which was noted in $41(24.8 \%)$ patients. A significant proportion (74.3\%) of isolates comprised of Gram negative bacilli while $22 \%$ were Gram positive. M. tuberculosis was responsible for 11 episodes of bacterial infections. Special pathogens such as Brucella spp., Burkholderia pseudomallei and Salmonella Typhi were isolated from two and one each patient respectively. E. coli was frequently isolated from ascitic fluid, blood and urine. RTI occurred more frequently during hospitalization with Klebsiella and Acinetobacter being responsible for most of these infections (Table II). It was noted that $78.8 \%(\mathrm{n}=41)$ of $E$. coli and $66.6 \%(\mathrm{n}=16)$ of Klebsiella were extended-spectrum $\beta$-lactamase (ESBL) producers. 
Table III. Univariate analysis for factors predicting mortality in cirrhotic patients.

\begin{tabular}{|c|c|c|c|c|}
\hline \multirow{2}{*}{ Variables } & & \multicolumn{2}{|c|}{ Outcome } & \multirow[b]{2}{*}{ p-value } \\
\hline & & $\begin{array}{c}\text { Survivors } \\
(n=120)(\%)\end{array}$ & $\begin{array}{c}\text { Non-survivors } \\
(\mathrm{n}=\mathbf{3 8})(\%)\end{array}$ & \\
\hline \multirow{2}{*}{ Type of infection } & Community & $94(78.4)$ & $26(21.6)$ & \multirow{2}{*}{0.001} \\
\hline & Hospital & $26(59.4)$ & $12(31.6)$ & \\
\hline \multirow{2}{*}{ Bacteremia } & Present & $34(70.83)$ & $14(28.6)$ & \multirow{2}{*}{0.376} \\
\hline & Absent & $96(80)$ & $24(20)$ & \\
\hline \multirow{2}{*}{ AFI } & Present & $51(69)$ & $23(31)$ & \multirow{2}{*}{0.053} \\
\hline & Absent & $69(82.8)$ & $15(17.2)$ & \\
\hline \multirow{2}{*}{ RTI } & Present & $19(61.3)$ & $12(36.7)$ & \multirow{2}{*}{0.073} \\
\hline & Absent & $101(79.6)$ & $26(20.4)$ & \\
\hline \multirow{2}{*}{ UTI } & Present & $26(93)$ & $2(7)$ & \multirow{2}{*}{0.021} \\
\hline & Absent & $94(72.3)$ & $36(27.7)$ & \\
\hline \multirow{3}{*}{ Child Pugh Category } & A & $6(100)$ & 0 & \multirow{3}{*}{$<0.001$} \\
\hline & B & $50(98)$ & $1(2)$ & \\
\hline & $\mathrm{C}$ & $64(63.3)$ & $37(36.7)$ & \\
\hline \multirow{2}{*}{ Acute kidney injury } & Present & $42(58.3)$ & $30(41.7)$ & \multirow[b]{2}{*}{$<0.001$} \\
\hline & Absent & $78(90.7)$ & $8(9.3)$ & \\
\hline \multirow{2}{*}{ Hepatic Encephalopathy } & Present & $22(48.9)$ & $23(51.1)$ & \multirow{2}{*}{$<0.001$} \\
\hline & Absent & $98(86.7)$ & $15(13.3)$ & \\
\hline \multirow{2}{*}{ UGI Bleed } & Yes & $12(60)$ & $8(40)$ & \multirow{2}{*}{0.075} \\
\hline & No & $102(77.3)$ & $30(22.7)$ & \\
\hline Albumin * & & $2.38 \pm 0.48$ & $2.24 \pm 0.53$ & 0.163 \\
\hline Creatinine $^{*}$ & & $1.48 \pm 0.98$ & $2.51 \pm 1.35$ & $<0.001$ \\
\hline Bilirubin $^{+}$ & & $3.8(1.8-6.2)$ & $6.2(3.3-12.3)$ & 0.004 \\
\hline
\end{tabular}

*Expressed as mean \pm SD, + expressed as median (Q1, Q3); UGI bleed: Upper gastrointestinal bleed; AFI: Ascitic fluid infection; RTI: Respiratory tract infection; UTI: Urinary tract infection.

Table IV. Multivariate analysis for factors predicting mortality in cirrhotic patients.

\begin{tabular}{|c|c|c|c|}
\hline Variable & p-value & $95 \% \mathrm{CI}$ & Adjusted Odds Ratio \\
\hline Type of infection & $\mathrm{p}=0.052$ & $0.11-1.01$ & 0.33 \\
\hline AFI & $\mathrm{p}=0.029$ & $1.11-7.12$ & 2.81 \\
\hline Hepatic encephalopathy & $\mathrm{p}<0.001$ & $0.070-0.422$ & 0.17 \\
\hline AKI & $\mathrm{p}<0.001$ & $0.077-0.502$ & 0.19 \\
\hline
\end{tabular}

CI: Confidence interval, AFI: Ascitic fluid infection, AKI: Acute kidney injury.

\section{Factors predicting mortality}

The overall in-hospital mortality was recorded in $38(24 \%)$ patients. The factors associated with mortality were type of infection, Child Pugh category, AKI, hepatic encephalopathy, UTI, creatinine and bilirubin levels. A high mortality rate was observed in patients with Child class C (36.7\%). The patients without UTI had a poorer outcome than the patients with UTI (p-value 0.021); however, the number of patients who died with UTI was small. The mean creatinine was high $(2.51 \pm 1.35)$ in the group of patients who died than in the group of patients with favorable outcome $(1.48 \pm 0.98)$ and the p-value was significant between the two groups ( $p$-value $<0.001$ ) (Table III). There was a significant difference in bilirubin value between the group of patients who had a favorable and poor outcome (p-value 0.004). Logistic regression analysis revealed that type of infection (OR: 0.33, 95\% CI: 0.11-1.01), AFI (OR: 2.81, 95\% CI: $1.11-$ 7.12), hepatic encephalopathy (OR: $0.17,95 \%$ CI: $0.070-$ 0.422) and AKI (OR: 0.19, 95\% CI: 0.077-0.502) were significantly associated with in-hospital mortality among cirrhotic patients (Table IV). 


\section{Discussion}

In the present study 193 episodes of bacterial infections were noted in 158 patients. 126 cases had single site infections whereas 32 had multiple site infections. The proportion of males was predominant $(90.5 \%$ vs. $9.5 \%$ females). The higher incidence of bacterial infections in males can be explained by the higher incidence of cirrhosis in them compared to females. Chronic alcohol intake was identified as a frequent etiology of cirrhosis in our study. It is believed that alcohol increases the permeability of the intestinal mucosa and decreases the activity of Kupffer cells, thus favoring infection $[14,15]$.

Ascitic fluid was the most common site of bacterial infection (38.3\%) similar to the observations recorded by Caly et al. and Preda et al $[7,14]$. As we know, the main trigger for SBP is bacterial translocation. The possible pathophysiology for bacterial translocation in patients with cirrhosis are intestinal bacterial overgrowth, altered intestinal permeability and altered gut-associated lymphoid tissue (GALT) immune response [16]. In our study, 24.8\% of patients presented with bacteremia and was the second most common site of infection which appears to be higher than in the previous studies conducted by Borzio $\mathrm{M}$ et al and Caly et al. where the incidence was $21 \%$ and $13.8 \%$ respectively $[7,17]$.

UTI occurred in $14.5 \%$ of patients, this is in discrepancy with other studies [7,17]. This difference may be explained by the fact that only culture positive cases were taken in the present study whereas in the other studies patients with leukocyturia alone were also included. It has been suggested that residual urinary volume and possible vesical dysfunction may be responsible for high incidence of UTI in cirrhotic patients especially in those with ascites [18]. RTIs were seen in $15.5 \%$ of patients, frequency of which is consistent with other studies [7,17]. This could be attributed to aspiration resulting from upper gastrointestinal bleeding and hepatic encephalopathy.

Positive cultures were obtained in 148 episodes of bacterial infections excluding CNNA. Gram negative bacteria constituted the largest group (74.3\%) than Gram positive isolates. The high incidence of gram negative isolates may be related to the enhanced translocation of bacterial flora from the gut into the bloodstream and ascitic fluid and is generally encountered in patients with decompensated cirrhosis $[17,6]$. We found that $E$. coli was frequently detected in patients with ascitic fluid infection, bacteremia and UTI which is similar to the results obtained by Fernandez et al [19]. Acinetobacter and Klebsiella were isolated more commonly from patients with nosocomial pneumonia while Mycobacterium tuberculosis was the commonest organism implicated in community acquired RTI in the present series. All the nine patients from whom Acinetobacter was isolated from the tracheal aspirate were on ventilator support. Streptococci were isolated in patients with community acquired pneumonia and pseudomonas in patients with hospital acquired pneumonia. This difference in the organisms causing community acquired RTI may be related to the low prevalence of tuberculosis in western population.

Patients with decompensated cirrhosis are more prone for bacterial infections accounting for about 30\%$50 \%$ deaths $[19,20]$. We observed that infections occurred more frequently in patients with advanced cirrhosis. This is probably due to increased immunosuppression which puts them at greater risk of acquiring infections. The mortality was noted in $24 \%$ of the patients which was almost similar to the mortality rates of $23.3 \%$ and $22 \%$ reported previously by Caly et al. and Borzio et al. respectively $[7,17]$, suggesting that mortality from bacterial infections in cirrhotics has not changed in the current era. The high mortality rate was observed in subjects with RTI (38.7\%) in the present study, this may be because majority of respiratory infections were hospital acquired and caused by multidrug resistant bacteria.

In a study conducted by Shih et al., in-hospital mortality was significantly high in patients with cirrhosis with bacteremia and was identified to be one of the independent risk factor for poor outcome (OD: 9.7; 95\% CI: 1.9-50.6) in the presence of gastrointestinal bleeding [21]. On the contrary, multivariate analysis in the present series identified AFI as a strong risk factor for reduced survival (OR: 2.81, 95\% CI: 1.11-7.12). Most of the infections in our study were community acquired, while more deaths were noted among patients with hospital acquired infections. This could be attributed to infection with resistant gram-negative organisms and decreased efficacy of empirical antibiotic treatment in these patients. This finding is in agreement with a previous study by Fernandez et al. which included 669 infections from two series (2005-2007 and 2010-2011), the hospital mortality rate of nosocomial infections ( $25 \%-48 \%$ respectively) was significantly higher than observed in community acquired episodes (7\%-21\%) [19]. It is therefore advisable to initiate appropriate antibiotics as early as possible according to the local epidemiological patterns in patients with hospital acquired infections.

Bacterial infections may also be a precipitating factor for hepatic encephalopathy and AKI. As described earlier, the possible mechanism is that bacterial infection induces excess cytokine production causing cardiovascular and endothelial dysfunction [22,23]. In our study, both hepatic encephalopathy $(\mathrm{P}<0.001)$ and AKI $(\mathrm{P}<0.001)$ showed significant association with mortality. We observed that more deaths occurred among patients without UTI compared to the cases with UTI with a significant $p$-value which may be explained by the presence of infection at the other site in these patients that might have contributed to mortality. 


\section{Conclusion}

Based on these results, we conclude that ascitic fluid was found to be the most predominant site of infection. Gram negative bacterial infections were more common, with Enterobacteriaceae group being responsible for most of the infections. The type of infection, hepatic encephalopathy, ascitic fluid infection and acute kidney injury had a significant association with in-hospital mortality. Since most of the cirrhotic patients with bacterial infection are asymptomatic, high degree of suspicion is critical to avert complications and in the presence of above mentioned factors, early initiation of appropriate antibiotics would prevent further deterioration of liver disease.

\section{Limitations}

There was selection bias where all patients during the study period were randomly selected and included. Only culture positive cases were taken except for culture negative variant of spontaneous ascitic fluid infection (CNNA). Subjects who had other evidence of infection with sterile cultures were not included in the study.

\section{References}

1. Tandon P, Garcia-Tsao G. Bacterial infections, sepsis, and multiorgan failure in cirrhosis. Semin Liver Dis. 2008;28:2642.

2. Arvaniti V, D'Amico G, Fede G, Manousou P, Tsochatzis $\mathrm{E}$, Pleguezuelo $\mathrm{M}$, et al. Infections in patients with cirrhosis increase mortality four-fold and should be used in determining prognosis. Gastroenterology. 2010;139:12461256.

3. Tuchendler E, Tuchendler PK, Madej G. Immunodeficiency caused by cirrhosis. Clin Exp Hepatol. 2018;4:158-164.

4. Bunchorntavakul C, Chamroonkul N, Chavalitdhamrong D. Bacterial infections in cirrhosis: A critical review and practical guidance. World J Hepatol. 2016;8:307-321.

5. Bonnel AR, Bunchorntavakul C, Reddy KR. Immune dysfunction and infections in patients with cirrhosis. Clin Gastroenterol Hepatol. 2011;9:727-738.

6. Fernández J, Navasa M, Gómez J, Colmenero J, Vila $\mathrm{J}$, Arroyo $\mathrm{V}$, et al. Bacterial infections in cirrhosis: epidemiological changes with invasive procedures and norfloxacin prophylaxis. Hepatology. 2002;35:140-148.

7. Caly WR, Strauss E. A prospective study of bacterial infections in patients with cirrhosis. J Hepatol. 1993;18:353358.

8. Brann OS. Infectious complications of cirrhosis. Curr Gastroenterol Rep. 2001;3:285-292.

9. Horan TC, Andrus M, Dudeck MA. CDC/NHSN surveillance definition of health care-associated infection and criteria for specific types of infections in the acute care setting. Am J Infect Control. 2008;36:309-332.
10. Runyon BA; Practice Guidelines Committee, American Association for the Study of Liver Diseases (AASLD). Management of adult patients with ascites due to cirrhosis. Hepatology. 2004;39:841-856.

11. CDC.CDC/NHSN protocol clarifications [Internet]. Georgia, Greater Atlanta: United States centres for disease control: 2019 [cited 2019 Jan]. Available from: www.cdc.gov/nhsn/ pdfs/pscmanual/7psccauticurrent.pdf

12. Bone RC, Balk RA, Cerra FB, Dellinger RP, Fein AM, Knaus WA, et al. Definitions for sepsis and organ failure and guidelines for the use of innovative therapies in sepsis. The ACCP/SCCM Consensus Conference Committee. American College of Chest Physicians/Society of Critical Care Medicine. Chest. 1992;101:1644-1655.

13. Ghany MG, Hoofnagle JH. Approach to the patient with liver disease. In: Longo DL, Fauci AS, Kasper DL, Hauser SL, Jameson JL, Loscalzo J. eds. Harrison's Principles of Internal Medicine. $20^{\text {th }}$ ed. USA: Mc Graw-Hill; 2018: pp. 2337.

14. Preda CM, Ghita R, Ghita C, Mindru C, Vlaicu L, Andrei A, et al. A retrospective study of bacterial infections in cirrhosis. Maedica (Buchar). 2011;6:185-192.

15. Rosa H, Silvério AO, Perini RF, Arruda CB. Bacterial infection in cirrhotic patients and its relationship with alcohol. Am J Gastroenterol. 2000;95:1290-1293.

16. Wiest R, Lawson M, Geuking M. Pathological bacterial translocation in liver cirrhosis. J Hepatol. 2014;60:197-209.

17. Borzio M, Salerno F, Piantoni L, Cazzaniga M, Angeli P, Bissoli $\mathrm{F}$, et al. Bacterial infection in patients with advanced cirrhosis: a multicentre prospective study. Dig Liver Dis. 2001;33:41-48.

18. Bercoff E, Déchelotte P, Weber J, Morcamp D, Denis P, Bourreille J. Urinary tract infection in cirrhotic patients, a urodynamic explanation. Lancet. 1985;1:987.

19. Fernández J, Acevedo J, Castro M, Garcia O, de Lope CR, Roca D, et al. Prevalence and risk factors of infections by multiresistant bacteria in cirrhosis: a prospective study. Hepatology. 2012;55:1551-1561.

20. Tandon P, Abraldes JG, Keough A, Bastiampillai R, Jayakumar S, Carbonneau M, et al. Risk of bacterial infection in patients with cirrhosis and acute variceal hemorrhage, based on child-pugh class, and effects of antibiotics. Clin Gastroenterol Hepatol. 2015;13:1189-1196.e2.

21. Shih HA, Tsai PC, Wu KH, Chen YT, Chen YC. Bacteremia in cirrhotic patients with upper gastrointestinal bleeding. Turk J Gastroenterol. 2018;29:164-169.

22. Jalan R, Fernandez J, Wiest R, Schnabl B, Moreau R, Angeli $P$, et al. Bacterial infections in cirrhosis: a position statement based on the EASL Special Conference 2013. J Hepatol. 2014;60:1310-1324.

23. Merli M, Lucidi C, Pentassuglio I, Giannelli V, Giusto M, Di Gregorio V, et al. Increased risk of cognitive impairment in cirrhotic patients with bacterial infections. J Hepatol. 2013;59:243-250. 